\title{
Construção do Aerofólio de Joukowski Assimétrico por meio da Transformação de Karman-Trefftz, para $n=2$, utilizando o GeoGebra 5.0
}

\author{
Construction of the Asymmetric Joukowski Airfoil through Karman-Trefftz Transformation, \\ for $n=2$, using GeoGebra 5.0
}

\begin{abstract}
João Jefferson Faria Marinho Escola Naval (EN), Departamento das Ciências Fundamentais, Rio de Janeiro, RJ, Brasil https://orcid.org/0000-0001-9986-3882, jefferson.en@hotmail.com
\end{abstract}

Raphael Francisco Gomes dos Santos Universidade Estácio de Sá (UNESA), Departamento de Engenharia Elétrica, Rio de Janeiro, RJ, Brasil https://orcid.org/0000-0002-1561-8503, raphaelfgsantos@gmail.com

Luciana Santos da Silva Martino Colégio Pedro II (CPII), Departamento de Matemática, Rio de Janeiro, RJ, Brasil https://orcid.org/0000-0002-4121-6292, lulismartino@gmail.com

\section{Informações do Artigo}

\section{Como citar este artigo}

MARINHO,João Jefferson Faria; SANTOS, Raphael Francisco Gomes dos; MARTINO, Luciana Santos da Silva. Construção do Aerofólio de Joukowski Assimétrico por meio da Transformação de Karman-Trefftz, para $n=2$, utilizando o GeoGebra 5.0. REMAT: Revista Eletrônica da Matemática, Bento Gonçalves, RS, v. 6, n. 2, p. e4001, 24 jul. 2020. DOI: https://doi.org/10. 35819/remat2020v6i2id3895
Resumo
A Transformação Conforme é uma ferramenta matemática que possibilita a construção de geometrias complexas a partir de geometrias mais simples. O aerofólio de Joukowski exemplifica essa afirmação, entretanto, a sua obtenção pelos meios matemáticos convencionais é complexa, tornando- se necessária a utilização de recursos computacionais. Neste traba- Iho é demonstrada uma técnica cujo propósito é facilitar o entendimento da Transformação de Joukowski, que consiste em mapear uma circun- ferência no aerofólio de Joukowski assimétrico com o uso do GeoGebra. A Transformação de Joukowski foi obtida por meio da Transformação de Karman-Trefftz, para $n=2$, fazendo uso de composições de funções com- plexas. As simulações foram realizadas utilizando o software GeoGebra 5.0, que possibilitou a construção e obtenção das equações matemáticas, no plano real, das figuras geométricas envolvidas nas transformações até a obtenção do aerofólio de Joukowski assimétrico. Os resultados da

\section{Palavras-chave}

Transformação de Joukowski

Transformação de Karman-Trefftz

Aerofólio de Joukowski Assimétrico

GeoGebra 


\section{Keywords}

Joukowski Transformation Karman-Trefftz Transformation Asymmetric Joukowski Airfoil GeoGebra

\begin{abstract}
The Conform Transformation is a mathematical tool that allows the construction of complex geometries from simpler geometries. The Joukowski airfoil exemplifies this statement, however, obtaining it by conventional mathematical means is complex, making it necessary to use computational resources. This article demonstrates a technique whose purpose is to facilitate the understanding of the Joukowski Transformation, which consists of mapping a circumference in the asymmetric Joukowski airfoil using GeoGebra. The Joukowski Transformation was obtained through the KarmanTrefftz Transformation, for $n=2$, using compositions of complex functions. The simulations were performed using the GeoGebra 5.0 software, which made it possible to construct and obtain mathematical equations, on the real plane, of the geometric figures involved in the transformations until obtaining the asymmetric Joukowski airfoil. The results of the simulation were satisfactory, such that the equations of the geometric shapes until the construction of the airfoil were successfully obtained.
\end{abstract}

\section{Introdução}

A Transformação de Joukowski é dada por

$$
w(z)=z+\frac{c^{2}}{z}, c>0
$$

em que $z \in \mathbb{C}$ e $c \in \mathbb{R}$. Nikolai Joukowski (1847-1921) foi um matemático russo que fez contribuições em aerodinâmica; sua Transformação tem relevância no estudo aerodinâmico de escoamentos potenciais, ganhando destaque na Matemática Aplicada. Essa transformação é capaz de mapear a circunferência em um aerofólio (ÁVILA, 2013). A Construção do aerofólio de Joukowski é árdua, como vista em Milne-Thompson (1996), e a dificuldade de encontrar literaturas que detalhem essa construção com clareza não é fácil, pois exige a aplicação de um software que consiga construir a geometria da figura. Desse modo, neste trabalho, será realizada a construção do aerofólio de Joukowski assimétrico utilizando o software GeoGebra 5.0, a partir da Transformação de Karman-Trefftz dada por

$$
\frac{w-2 c}{w+2 c}=\left(\frac{z-c}{z+c}\right)^{2}
$$

para $n=2$.

Essa transformação possibilita a construção de 3 funções e, a partir delas, torna-se possível a realização de composições que levam à construção do aerofólio. O GeoGebra é um software livre e apresenta ferramentas para construções geométricas dinâmicas. Com a utilização do GeoGebra é possível obter as equações das formas geométricas envolvidas, por meio da manipulação de controles dinâmicos construídos. 


\section{Metodologia}

2.1 Transformação de Joukowski a partir da Transformação de Karman-Trefftz, para $n=2$

Neste trabalho pretende-se construir o aerofólio de Joukowski assimétrico mapeando uma circunferência do plano $-z$ para $\circ$ plano $-z_{1}$, mapear a curva obtida nesse plano para o plano $-z_{2}$ e por fim mapear a curva obtida nesse plano para o plano-w, obtendo assim o aerofólio. Outros aspectos poderiam ser analisados, como por exemplo, como são mapeados os pontos exteriores à circunferência usando essas transformações.

Uma transformação $T: D \rightarrow K$ é conforme quando $T$ é bijetora e analítica, em que $D$ e $K$ são regiões do plano complexo. Desse modo, a transformação $T$ preserva ângulos. As Transformações Conformes são técnicas para solucionar problemas em áreas como aerodinâmica e hidrodinâmica, pois usando a transformação adequada é possível estudar escoamentos em regiões teoricamente mais simples e, por meio disso, entender o escoamento em regiões mais complexas.

A Transformação de Karman-Trefftz, para $n=2$, dada pela equação (2), com $c \in \mathbb{R}$, é uma transformação conforme (KYTHE, 1998). Dessa transformação podemos construir 3 transformações

$$
z=T(w)=\frac{w-2 c}{w+2 c}, z_{1}=T_{1}(z)=\frac{z-c}{z+c} \text { e } z_{2}=T_{2}\left(z_{1}\right)=z_{1}^{2},
$$

onde cada uma delas é conforme dentro das suas permissividades de domínio.

Como $T$ é conforme, a sua inversa existe e também é conforme (ABLOWITZ; FOKAS, 2003). Portanto, isso nos permite construir a Transformação de Joukowski a partir da composição

$$
T^{-1}\left(T_{2}\left(T_{1}(z)\right)\right)
$$

onde $T^{-1}(z)=-2 c \frac{z+1}{z-1}$.

\subsection{O GeoGebra}

O GeoGebra é um laboratório matemático com ferramentas dinâmicas adequadas para o ensino e a aprendizagem da matemática e sua distribuição é gratuita por meio do site www.geogebra.org, onde são encontras algumas versões do software. O GeoGebra possui uma interface intuitiva, o que possibilita ao usuário usá-lo com facilidade, mesmo que para iniciantes. Permite a criação de construções matemáticas e modelos que possibilitam explorações interativas e dinâmicas com mudanças de perspectivas, o que contribui, segundo Basniak e Estevam (2014), para o desperta- 
mento da intuição e da maneira de visualizar um problema em diversos níveis de ensino. Criado em 2001 por Markus Hohenwarter, na Universidade de Salzburgo, o GeoGebra tem como objetivo, segundo Hall e Lingefjärd (2017), fornecer gratuitamente aos alunos de Matemática a possibilidade da realização de pesquisas investigativas nas áreas da Geometria, Aritmética, Álgebra, Números Complexos, entre outras.

O argumento de Baldin (2002, p. 32),

Os programas de Geometria Dinâmica (DGS) [...] possuem qualidades como as de visualização e de tela interativa para explorar propriedades geométricas, podem ser utilizados, com eficiência, como auxiliar na construção de raciocínio dedutivo e de capacidade especulativa, podem ser auxiliares na modelagem de problemas com simulações, etc.

adequa-se ao GeoGebra. O software dispõe de janelas que permitem ao usuário visualizações de objetos 2D e 3D potencializando a capacidade de investigar situações-problemas da Matemática e apresentando uma solução visual do problema. Como exemplo, podemos citar a planificação e rotação de uma pirâmide, as construções de lugares geométricos e, em nosso caso, a construção do aerofólio de Joukowski assimétrico.

Como uma representação simples, a Figura 1 demonstra o ambiente gráfico de modelagem e simulação no software GeoGebra para a planificação de uma pirâmide.

Figura 1: Modelagem de uma pirâmide no GeoGebra 5.0. No software é possível visualizar a dinâmica da planificação da pirâmide com o controle deslizante na janela de trabalho.

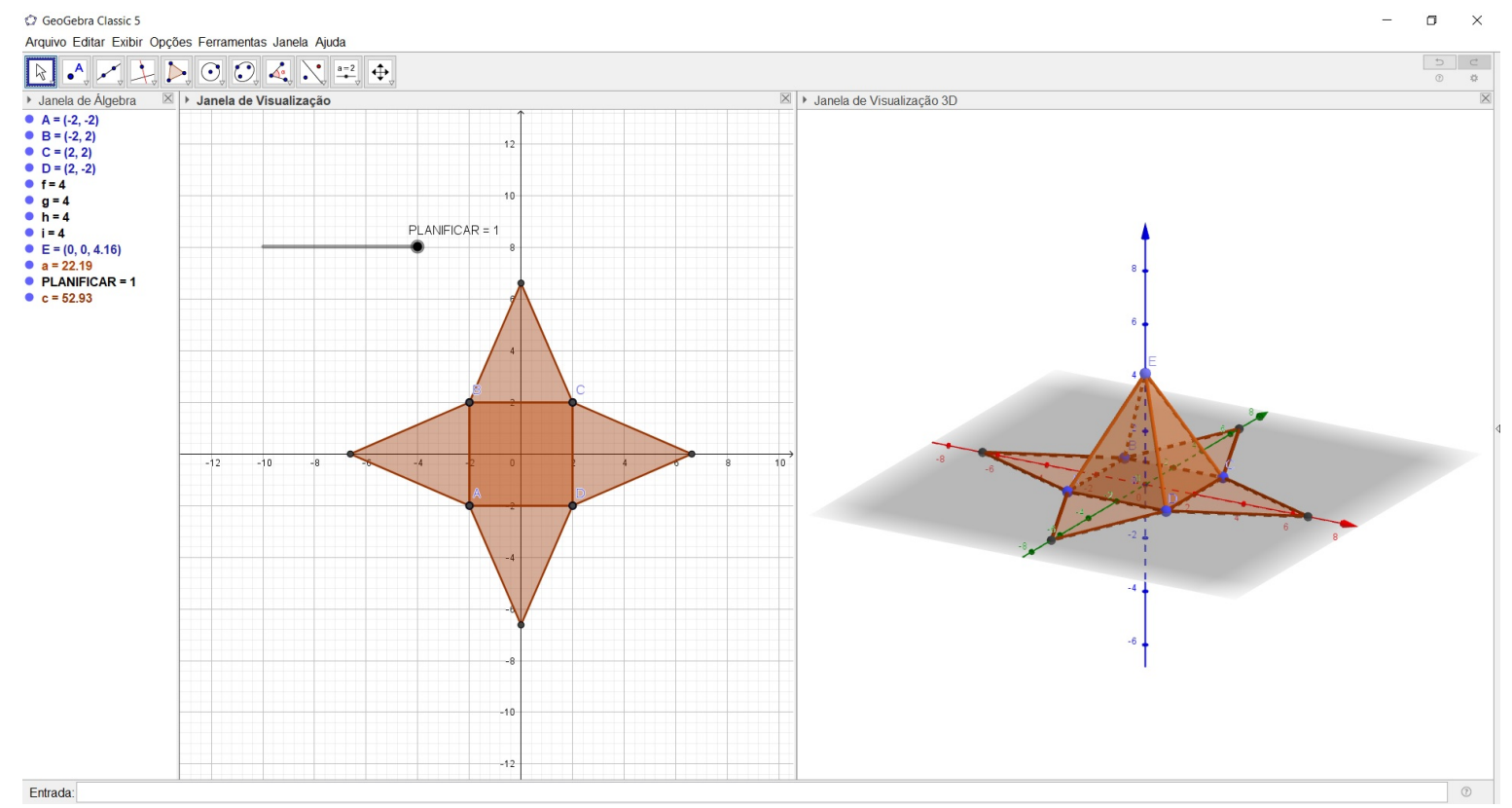

Fonte: Elaboração dos autores (2020). 


\subsection{Observações Importantes para a Construção}

O GeoGebra só visualiza uma entrada para cada coordenada. Como será usada a janela 2D, as partes real e imaginária de cada transformação (3) será vista como uma entrada para cada coordenada nesse software. Assim, destacamos as seguintes observações:

(a) Uma função complexa $f$ é definida por $f(z)=u(x, y)+i v(x, y)$.

(b) O número complexo $z$ é dado por $z=x+i y$, $\operatorname{com} x, y \in \mathbb{R}$.

(c) A construção das partes real e imaginária da função complexa serão representadas pelas coordenadas $x$ e $y$ do GeoGebra, respectivamente, mesmo $u$ e $v$ sendo bidimensionais.

(d) Para cada transformação (3) deve ser feita a separação das partes real e imaginária.

(e) O valor da constante $c$ apresentado na Transformação de Karman-Trefftz será igual a 1.

Para elucidar essas observações, usaremos a transformação $T_{2}\left(z_{1}\right)=z_{1}^{2}$ no plano- $z_{2}$. Ao separar as partes real e imaginária obtemos $T_{2}\left(z_{1}\right)=x_{1}^{2}-y_{1}^{2}+i 2 x_{1} y_{1}$. A parte real $x_{1}^{2}-y_{1}^{2}$ será a coordenada $x$ e a imaginária $2 x_{1} y_{1}$ será a coordenada $y$ no GeoGebra. Os valores de entrada para $x_{1}$ e $y_{1}$ serão os valores de $x$ e $y$ da curva anterior representados no GeoGebra.

\subsection{Construção do Aerofólio de Joukowski}

Primeiramente, constroem-se 4 controles deslizantes: parâmetro $t$, raio $R$, parte real $a$ e parte imaginária $b$ do número complexo $z_{0}=a+i b$. O ícone do controle deslizante pode ser visualizado na Figura $2 \mathrm{a}$.

Figura 2: Interface do GeoGebra.

(a) O controle deslizante é uma ferramenta que permite dinamizar, manualmente ou automaticamente, um objeto.

2 GeoGebra Classic 5

Arquivo Editar Exibir Opções Ferramentas Janela Ajuda

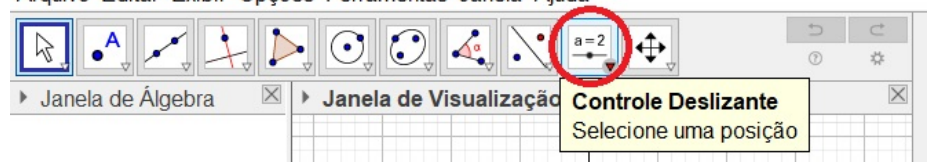

(b) Local onde são inseridos os comandos manualmente.

Fonte: Elaboração dos autores (2020). 
No local ENTRADA, Figura 2b, digitamos o comando Curva para construir uma equação paramétrica de uma circunferência $C$ de centro em $z_{0}=-0.209+i 0.2737$ e raio $R=1.2395$, onde $t \in[0,2 \pi]$ é o parâmetro para a variação de $\theta$ (Figura 3). Assim, o comando a ser inserido é

$$
\text { Curva }(R \cos (\theta)+a, R \operatorname{sen}(\theta)+b, \theta, 0, t) .
$$

Figura 3: Circunferência de centro em $z_{0}=-0.209+i 0.2737$ e raio $R=1.2395$.

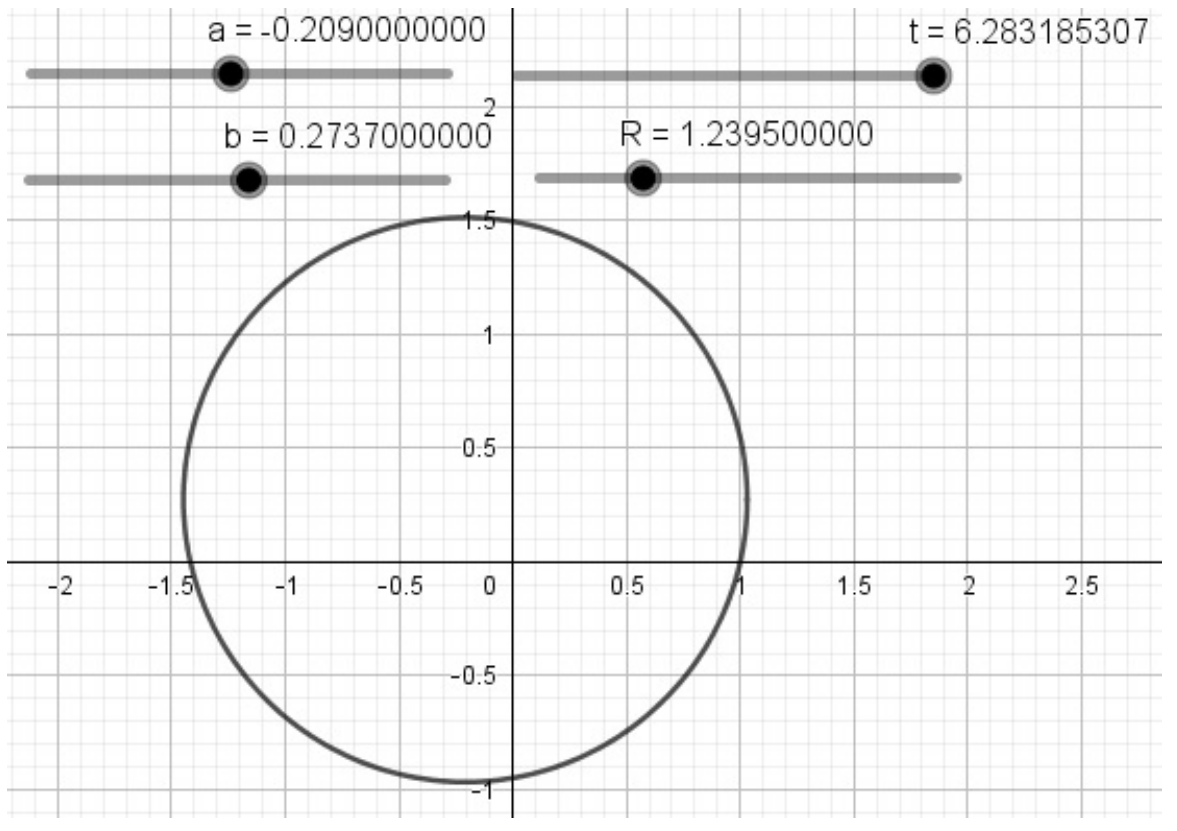

Fonte: Elaboração dos autores (2020).

Usando a transformação $T_{1}(z)$ e fazendo a separação entre as partes real e imaginária, obtemos

$$
T_{1}(z)=\frac{x^{2}+y^{2}-1}{(x+1)^{2}+y^{2}}+i \frac{2 y}{(x+1)^{2}+y^{2}} .
$$

Agora, devemos substituir os valores de $x$ e $y$ da transformação (5) pelos da construção da circunferência $C$, isto é, $x=R \cos (\theta)+a \mathrm{e} y=R \operatorname{sen}(\theta)+b$. Feito isso, o comando no local ENTRADA será

$$
\text { Curva }\left(\frac{(R \cos (\theta)+a)^{2}+(R \operatorname{sen}(\theta)+b)^{2}-1}{(R \cos (\theta)+a+1)^{2}+(R \operatorname{sen}(\theta)+b)^{2}}, \frac{2(R \operatorname{sen}(\theta)+b)}{(R \cos (\theta)+a+1)^{2}+(R \operatorname{sen}(\theta)+b)^{2}}, \theta, 0, t\right) .
$$

A Figura 4 representa o mapeamento da curva $C$ no plano- $z_{1}$. 
Figura 4: Curva $C_{1}$ obtida pelo mapeamento da curva $C$ pela transformação $T_{1}(z)$.

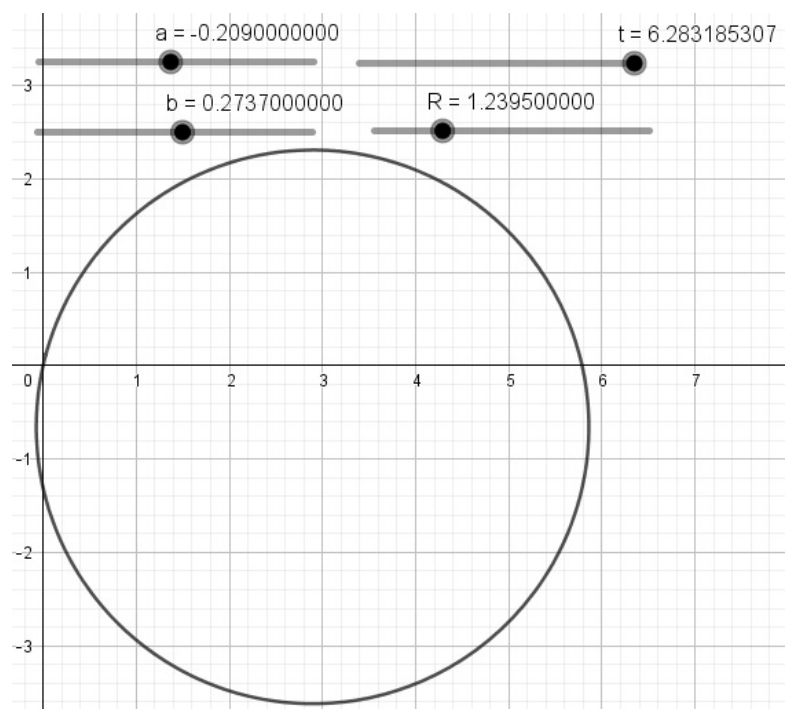

Fonte: Elaboração dos autores (2020).

O próximo passo é feito de modo análogo. Usando a segunda composição e a escrevendo em função de $x_{1}$ e $y_{1}$, obtemos que $T_{2}\left(z_{1}\right)=x_{1}^{2}-y_{1}^{2}+i 2 x_{1} y_{1}$, o que nos permite escrever

$$
T_{2}\left(T_{1}(z)\right)=\left(\frac{x^{2}+y^{2}-1}{(x+1)^{2}+y^{2}}\right)^{2}-\left(\frac{2 y}{(x+1)^{2}+y^{2}}\right)^{2}+i 2\left(\frac{x^{2}+y^{2}-1}{(x+1)^{2}+y^{2}}\right)\left(\frac{2 y}{(x+1)^{2}+y^{2}}\right),
$$

onde os valores de $x$ e $y$ a serem substituídos são os da curva $C$.

Figura 5: Curva $C_{2}$ obtida pelo mapeamento da curva $C_{1}$ pela transformação $T_{2}\left(z_{1}\right)$.

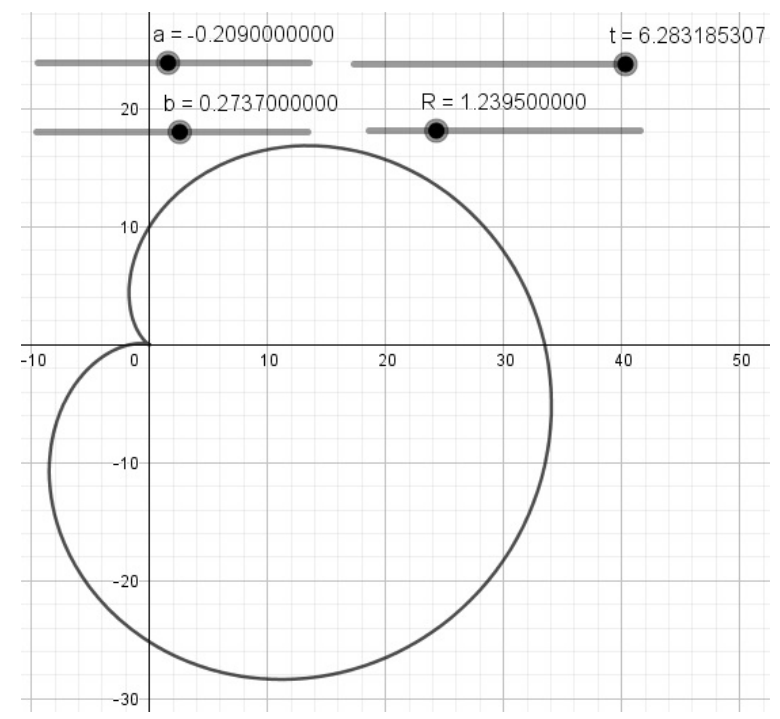

Fonte: Elaboração dos autores (2020).

A Figura 5 representa a curva obtida pelo mapeamento da curva $C_{1}$ pela transformação $T_{2}\left(T_{1}(z)\right)$ quando o comando inserido na ENTRADA é 


$$
\begin{aligned}
\text { Curva } & \left(\left(\frac{(R \cos (\theta)+a)^{2}+(R \operatorname{sen}(\theta)+b)^{2}-1}{(R \cos (\theta)+a+1)^{2}+(R \operatorname{sen}(\theta)+b)^{2}}\right)^{2}-\left(\frac{2(R \operatorname{sen}(\theta)+b)}{(R \cos (\theta)+a+1)^{2}+(R \operatorname{sen}(\theta)+b)^{2}}\right)^{2},\right. \\
& 2\left(\frac{(R \cos (\theta)+a)^{2}+(R \operatorname{sen}(\theta)+b)^{2}-1}{(R \cos (\theta)+a+1)^{2}+(R \operatorname{sen}(\theta)+b)^{2}}\right)\left(\frac{2(R \operatorname{sen}(\theta)+b)}{(R \cos (\theta)+a+1)^{2}+(R \operatorname{sen}(\theta)+b)^{2}}\right) \\
& \theta, 0, t)
\end{aligned}
$$

Por fim, fazendo a composição da transformação

$$
T^{-1}\left(z_{2}\right)=-2\left(\frac{x_{2}^{2}+y_{2}^{2}-1}{\left(x_{2}-1\right)^{2}+y_{2}^{2}}-i \frac{2 y_{2}}{\left(x_{2}-1\right)^{2}+y_{2}^{2}}\right)
$$

com $T_{2}\left(T_{1}(z)\right)$ e agindo de modo análogo ao caso anterior, obtemos o aerofólio de Joukowski assimétrico (Figura 6), quando o valor de ENTRADA a ser inserido é

$$
\text { Curva }\left(\left(\frac{A^{2}+B^{2}-1}{(A-1)^{2}+B^{2}}\right), \frac{2 B}{(A-1)^{2}+B^{2}}, \theta, 0, t\right)
$$

onde $A=\left(\frac{(R \cos (\theta)+a)^{2}+(R \operatorname{sen}(\theta)+b)^{2}-1}{(R \cos (\theta)+a+1)^{2}+(R \operatorname{sen}(\theta)+b)^{2}}\right)^{2}-\left(\frac{2(R \operatorname{sen}(\theta)+b)}{(R \cos (\theta)+a+1)^{2}+(R \operatorname{sen}(\theta)+b)^{2}}\right)^{2}$ e $B=$ $2\left(\frac{(R \cos (\theta)+a)^{2}+(R \operatorname{sen}(\theta)+b)^{2}-1}{(R \cos (\theta)+a+1)^{2}+(R \operatorname{sen}(\theta)+b)^{2}}\right)\left(\frac{2(R \operatorname{sen}(\theta)+b)}{(R \cos (\theta)+a+1)^{2}+(R \operatorname{sen}(\theta)+b)^{2}}\right)$.

Figura 6: Aerofólio de Joukowski assimétrico obtido pelo mapeamento da curva $C_{3}$ pela transformação $T^{-1}\left(z_{2}\right)$.

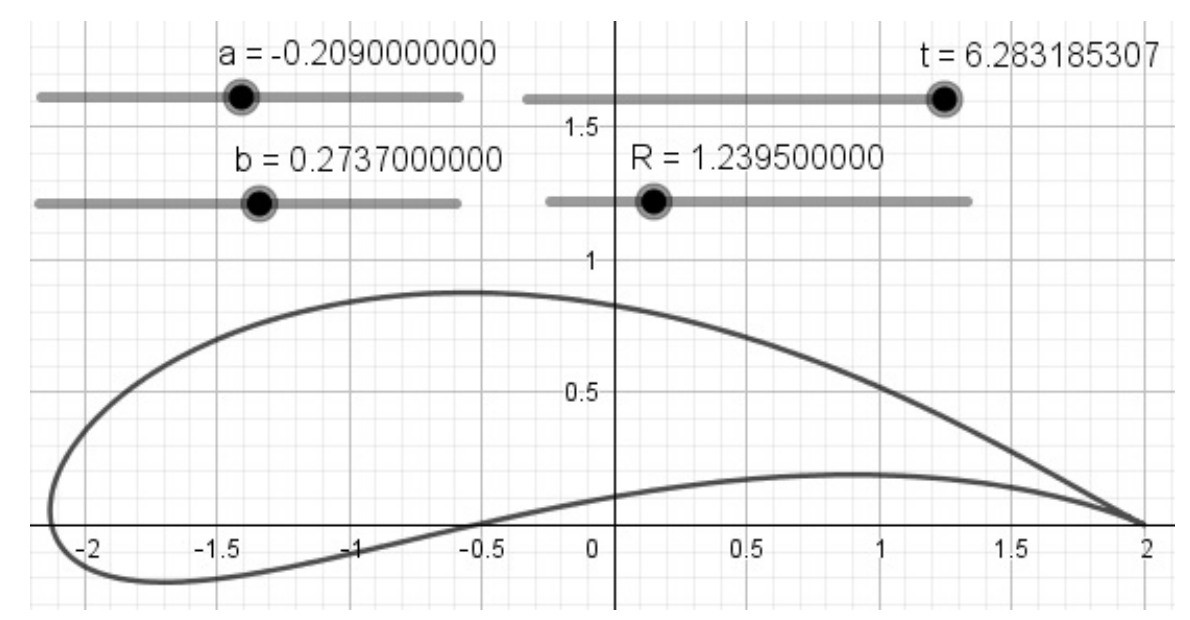

Fonte: Elaboração dos autores (2020).

\section{Resultados}

Para os resultados adquiridos, admitimos um trucamento de 4 casas decimais e desprezamos o erro cometido. 
Inicialmente, utilizou-se o GeoGebra 6.0. No entanto, esse software apresentou dificuldades ao serem digitados os códigos, na entrada, devido sua janela de digitação curta, lentidão e travamento nas execuções dos comandos, mostrando-se instável para a simulação. O GeoGebra 5.0 mostrou-se satisfatório para a simulação, permitindo as construções sem apresentar erros da sua versão superior.

Com um truncamento de 4 casas decimais, observa-se de forma satisfatória as construções apresentadas nas Figuras 3, 4, 5, e 6 quando comparadas com as dos autores Vallentine (1967), Milne-Thompson (1996), Batchelor (2000), Acheson (2005) e Pontes e Mangiavacchi (2016). Porém, em uma aproximação em torno dos valores de $x \in[1.99897,1.99903]$ e do ponto $(2,0)$, é possível visualizar os erros cometidos pelo truncamento. A Figura $7 \mathrm{a}$ apresenta um ponto de interseção para um valor de $x$ no intervalo citado e a Figura $7 \mathrm{~b}$ mostra que o bordo de fuga do aerofólio apresenta uma forma boleada, quando na verdade deveria ter o formato de uma cúspide (BATCHELOR, 2000), e a distância da origem, medida no eixo $x$, até esse bordo de fuga, mede um valor maior que 2. Entretanto, segundo Pope (1951), essa distância deve medir $2 c$; como definimos $c=1$, essa distância deveria ser 2.

Esses erros são sanados quando tomamos o raio igual ao número irracional

$$
R=\sqrt{(1.209)^{2}+(0.2737)^{2}}
$$

porém, no GeoGebra 5.0, não é possível fazer essa verificação devido a suas limitações na dinâmica de aproximação em torno do ponto $(2,0)$.

Portanto, após as simulações de cada transformação (3) no GeoGebra 5.0, os resultados obtidos foram:

a) A transformação $T_{1}(z)$ mapeia $C$ em uma circunferência $\left|z_{1}-(2.8911-i 0.6543)\right|=8.7865$ no plano $-z_{1}$.

b) A transformação $T_{2}\left(T_{1}(z)\right)$ mapeia $C_{1}$ em um cardioide, $C_{2}$, no plano- $z_{2}$, cuja equação pode ser dada por $r=17.5976(1+\cos (\theta-5.8434))$.

c) A transformação $T^{-1}\left(T_{2}\left(T_{1}(z)\right)\right)$ mapeia o cardioide no aerofólio de Joukowski assimétrico no plano- $w$.

d) Conseguimos construir uma dinâmica que, além de permitir visualizar as transformações das curvas geradas pelas transformações (3), permite visualizar outros tipos de figuras geométricas que têm aplicações em Dinâmica de Fluidos de escoamentos potenciais, como vistas em 
Vallentine (1967), Milne-Thompson (1996), Batchelor (2000), Acheson (2005) e Pontes e Mangiavacchi (2016) e mostradas na Figura 8.

Figura 7: Erros cometidos pelo truncamento de 4 casas decimais na construção do aerofólio.

(a) Erro cometido no intervalo [1.99897, 1.99903].

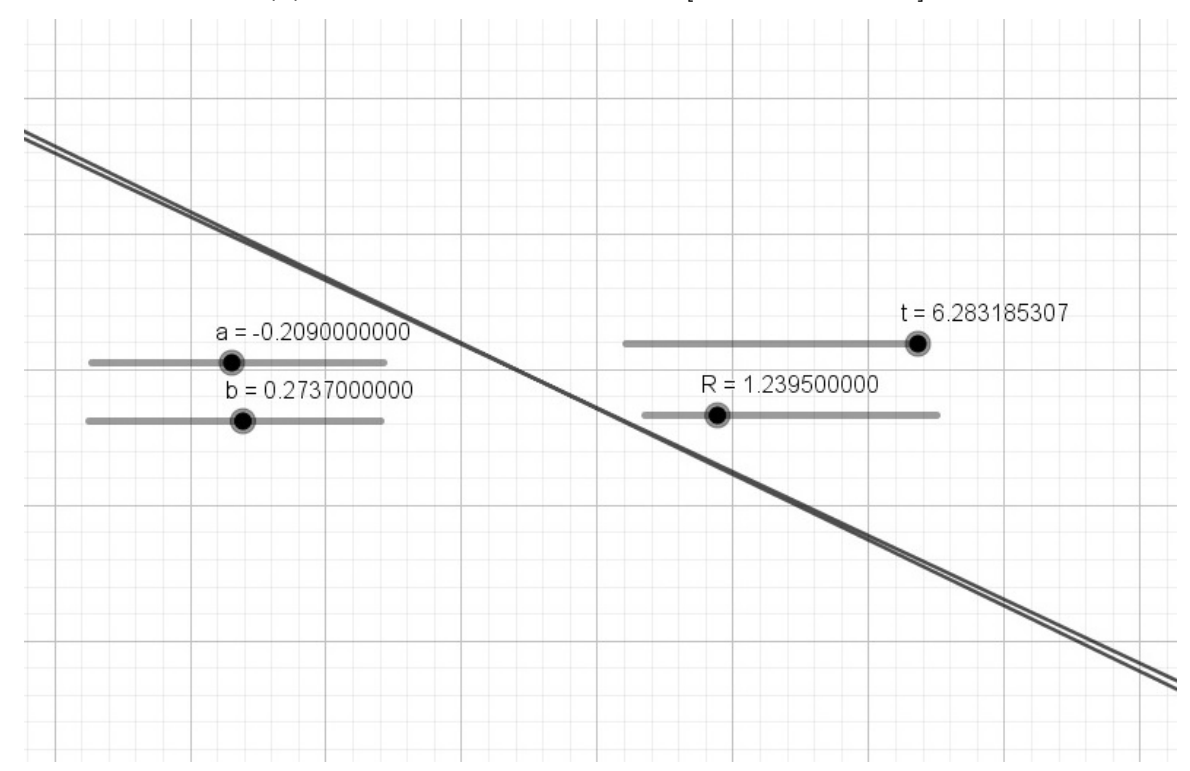

(b) Erro cometido no bordo de fuga.

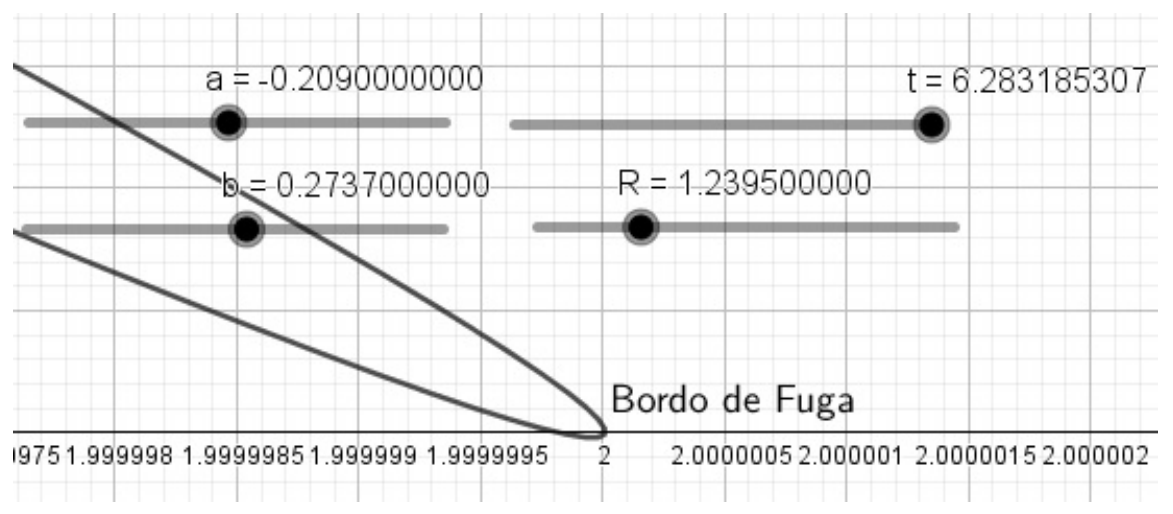

Fonte: Elaboração dos autores (2020). 
Figura 8: Imagens de circunferências obtidas pela Transformação de Joukowski, em que $z_{0}=a+i b$ e $R$ são, respectivamente, o centro e o raio das circunferências, $\operatorname{com} c=1$. Dados retirados de Pontes e Mangiavacchi (2016), com exceção do aerofólio assimétrico (Figura 8d).

(a) $z_{0}=0$ e $R=1$.

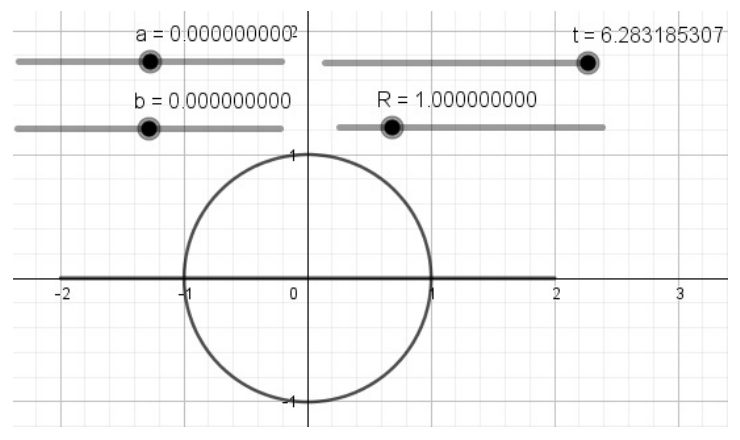

(c) $z_{0}=-0.2$ e $R=1.2$.

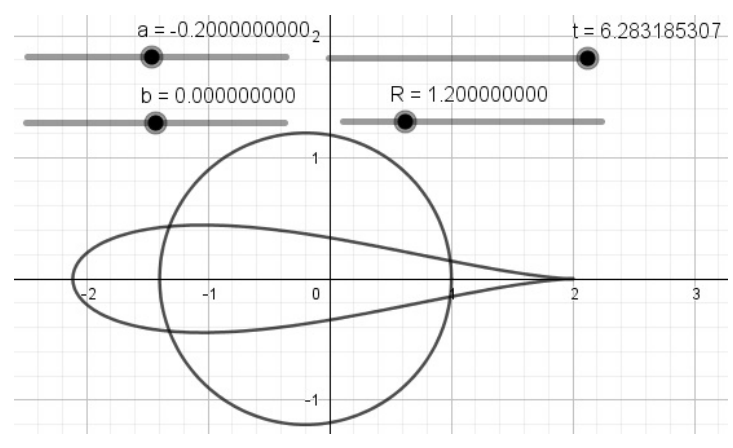

(b) $z_{0}=0$ e $R=1.3250$.

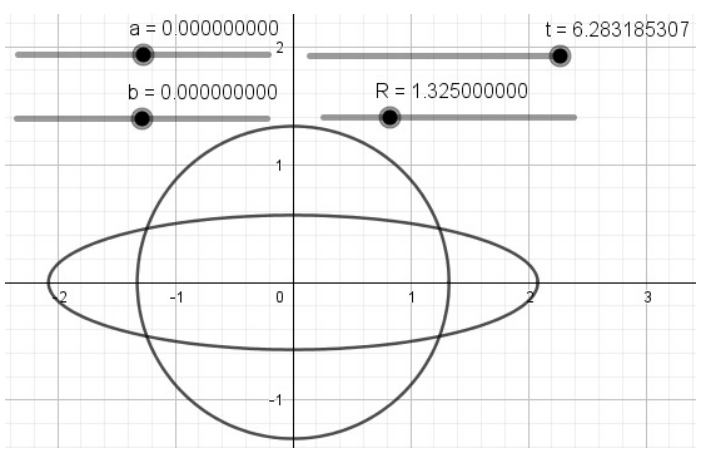

(d) $z_{0}=-0.209+i 0.2737$ e $R=1.2395$.

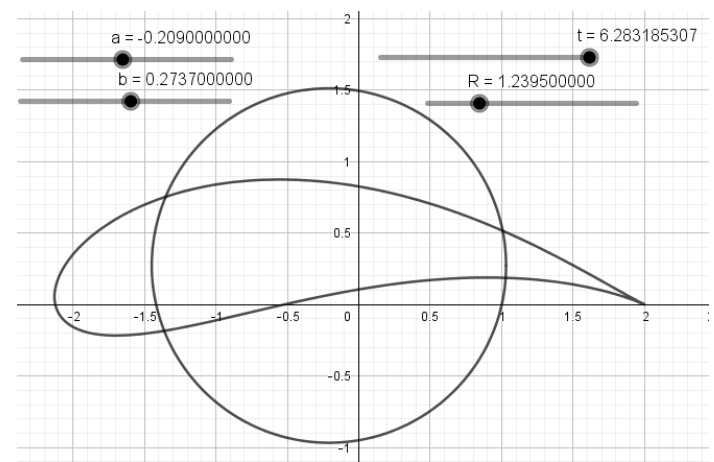

Fonte: Elaboração dos autores (2020).

\section{Conclusão}

A utilização do GeoGebra 5.0 no mapeamento da Transformação de Joukowski, por meio da Transformação de Karman-Trefftz, para $n=2$, demonstrou ser uma ferramenta adequada para a modelagem matemática do aerofólio. O truncamento de 4 casas decimais, desprezando-se o erro cometido, mostrara-se adequado, pois a imagem obtida foi considerada satisfatória quando comparada com as imagens dos aerofólios apresentadas por Vallentine (1967), Milne-Thompson (1996), Batchelor (2000), Acheson (2005) e Pontes e Mangiavacchi (2016). A construção permitiu, também, a visualização do comportamento da Transformação de Joukowski quando é realizada a mudança do raio e do centro da circunferência inicial, o que permitiu obter outros resultados sobre as curvas geradas por essa transformação (Figura 8).

Este trabalho possibilita a visualização da expansão da aplicação do software, a saber, como construir a visualização dos pontos exteriores a uma circunferência quando mapeadas pela 
Transformação de Joukowski. Esse entendimento deve ser considerado pertinente, pois pode-se modelar o escoamento de um fluido em torno de um cilindro circular usando Funções Complexas. Essa modelagem é realizada em uma vista bidimensional, assim, a fronteira do cilindro circular é interpretada como uma circunferência. Dessa forma, podemos entender o comportamento do fluido exterior a um aerofólio entendendo o comportamento do fluido exterior a um cilindro circular.

\section{Referências}

ABLOWITZ, M. J.; FOKAS, A. S. Complex Variables Introduction and Applications. 2. ed. New York: Cambridge, 2003. p. 342-343.

ACHESON, D. J. Elementary Fluid Dynamics. 1. ed. Oxford, New York: Clarendon Press, 2005. p. 136-140.

ÁVILA, G. Variáveis Complexas e Aplicações. 3. ed. Rio de Janeiro: LTC, 2013. p. 235-238.

BALDIN, Y. Y. Utilizações diferenciadas de recursos computacionais no ensino de Matemática (CAS, DGS e Calculadoras Gráficas). In: COLÓQUIO DE HISTÓRIA E TECNOLOGIA NO ENSINO DE MATEMÁTICA, 1., 2002, Rio de Janeiro. Anais... Rio de Janeiro: UERJ, p. 27-36, 2002.

BASNIAK, Maria Ivete; ESTEVAM, Everton José Goldoni. O GeoGebra e a Matemática da Educação Básica: frações, estatística, círculo e circunferência. 1. ed. Curitiba: Íthala, 2014. p. 13.

BATCHELOR, G. K. An Introduction to Fluid Dynamics. 1. ed. New York: Cambridge, 2000.

HALL, Jonas; LINGEFJÄRD, Thomas. Mathematical Modeling Applications with GeoGebra. 1. ed. Nova Jersey: Wiley, 2017.

KYTHE, Prem K. Computational Conformal Mapping. 1. ed. Danvers: Biräuser Boston, 1998.

MILNE-THOMPSON, L. M. Theoretical Hydrodynamics. 5. ed. New York: Dover, 1996.

PONTES, J.; MANGIAVACCHI, N. Fenômenos de Transferência com Aplicações às Ciências Físicas e à Engenharia. 1. ed. Rio de Janeiro: SBM, 2016.

POPE, A. Basic Wing and Airfoil Theory. 5. ed. New York: McGraw-Hill, 1951.

VALLENTINE, H. R. Applied Hydrodynamics. 2. ed. New York: Springer, 1967. 